\title{
A FIXED POINT THEOREM FOR SOME NON-SELF-MAPPINGS
}

\author{
NADIM A. ASSAD
}

\begin{abstract}
A bstract. A fixed point theorem is proved for continuous mappings from a nonempty closed subset $K$, of a Banach space $X$, into $X$, and which satisfies contractive definition definition (3) and property (a) below.
\end{abstract}

\section{The Main Theorem.}

The following result was established in [5]: Let $X$ be a Banach space, $K$ a nonempty closed subset of $X$. Let $T: K \rightarrow X$ satisfy the following contractive condition on $K$ :

There exists a constant $h, 0<h<1$ such that, for each $x, y \in K$,

$$
\text { (1) } d(T x, T y) \leq h \max \cdot\{d(x, y) / 2, \quad d(x, T x), d(y, T y),[d(x, T y)+d(y, T x)] / q\},
$$

where $q$ is any real number satisfying $q \geq 1+2 h$. Suppose that $T$ has the additional property:

(a) for each $x \in \partial K$, the boundary of $K, T x \in K$, then $T$ has a unique fixed point. follows:

In proving his theorem [5], Rhoades constructed two sequences $\left\{x_{n}\right\}$ and $\left\{x_{n}^{\prime}\right\}$ as

Definition. Let $x_{0} \in K$. Define $x_{1}^{\prime}=T x_{0}$. If $x_{1}^{\prime} \in K$, set $x_{1}=x_{1}^{\prime}$. If $x_{1}^{\prime} \notin K$, choose $x_{1} \in \partial K$ so that $d\left(x_{0}, x_{1}\right)+d\left(x_{1}, x_{1}^{\prime}\right)=d\left(x_{0}, x_{1}^{\prime}\right)$. Set $x_{2}^{\prime}=T x_{1}$. If $x_{2} \in K$, set $x_{2}=x_{2}^{\prime}$. If not, choose $x_{2} \in \partial K$ so that $d\left(x_{1}, x_{2}\right)+d\left(x_{2}, x_{2}^{\prime}\right)=d\left(x_{1}, x_{2}^{\prime}\right)$. Continuing in this manner, we obtain $\left\{x_{n}\right\},\left\{x_{n}^{\prime}\right\}$ satisfying:

(i) $x_{n+1}^{\prime}=T x_{n}$,

(ii) $x_{n}=x_{n}^{\prime}$ if $x_{n}^{\prime} \in K$, and

(iii) $x_{n} \in \partial K$ and $d\left(x_{n-1}, x_{n}\right)+d\left(x_{n}, x_{n}^{\prime}\right)=d\left(x_{n-1}, x_{n}^{\prime}\right)$ if $x_{n}^{\prime} \notin K$.

Let $P=\left\{x_{i} \in\left\{x_{n}\right\}: x_{i}=x_{i}^{\prime}\right\}$ and $Q=\left\{x_{i} \in\left\{x_{n}\right\}: x_{i} \notin x_{i}^{\prime}\right\}$. The sequence $\left\{x_{n}\right\}$ will be referred to as the general orbit of $T$ at $x_{0}$.

Rhoades [5], proceeded in his proof by showing that for any $x_{0} \in K$, the general orbit of $T$ at $x_{0}$ is a Cauchy sequence that converges to the unique fixed point of $T$. On

Received October 5, 1989, reviewed February 22, 1990.

A.MS(1980) Subject classification. Primary 54H25; Secondary $47 \mathrm{H} 10$.

Key words and phrases. Banach space, fixed point. 
the other hand, in [3], the author has shown that if we require $T$ to be continuous and $K$ compact then we may replace condition (1) on $T$ by the following weaker condition:

$$
\text { For all : } x, y \in K, x \neq y \text {, }
$$

$$
d(T x, T y)<\max .\{d(x, y) / 2, d(x ; T y), d(y, T y),[d(x, T y)+d(y, T x)] / q\}
$$

where $q \geq 3$ and still conclude that $T$ has a unique fixed point.

In this paper, we prove a fixed point theorem for the mapping $T$ satisfying (a) and the following condition:

Let $R^{+}$denote the set of non-negative real numbers and let $h: R^{+} \backslash\{0\} \rightarrow(0,1)$ be a, decreasing function. Suppose that for all $x \neq y, x, y \in K$ :

(3) $d(T x, T y) \leq h(d(x, y)) \cdot \max \cdot\{d(x, y) / 2, d(x, T x), d(y, T y),[d(x, T y)+d(y, T x)] / q\}$,

where $q$ is any real number satisfying $q \geq 1+2 h(d(x, y))$. Observe that the above three conditions on $T$ are related as follows : (1) $\Rightarrow(3) \Rightarrow(2)$.Our results show that general orbit for the mapping $T$ satisfying (a) and (3) at any point $x_{0} \in K$ is a Cauchy sequence. Moreover, under the additional assumption that $T$ is continuous we may conclude that this Cauchy sequence converges to a unique fixed point of $T$.

Theorem. Let $X$ be a Banach space, $K$ a nonempty closed subset of $X, T: K \rightarrow X$ a continuous mapping satisfying (3) on $K$. If $T$ has property (a) then $T$ has a unique fixed point in $K$.

Proof. We will use the following notation: $\tau_{n}=d\left(x_{n}, x_{n+1}\right)$ and $s_{n}=d\left(x_{n}, x_{n+2}\right)$. It is easy to see that $s_{n}>0$ for each $n$. Moreover, following the proof of (Theorem $3.1,[2])$ we may assume that $\tau_{n}>0$ for each $n$.

Step I: We first wish to estimate $d\left(x_{n}, x_{n+1}\right)$.

Case I. $\quad x_{n}, x_{n+1} \in P$.

$$
\begin{aligned}
d\left(x_{n}, x_{n+1}\right)= & d\left(T x_{n-1}, T x_{n}\right) \\
\leq & h\left(d\left(x_{n-1}, x_{n}\right)\right) \cdot \max \cdot\left\{d\left(x_{n-1}, x_{n}\right) / 2, d\left(x_{n-1}, T x_{n-1}\right),\right. \\
& \left.d\left(x_{n}, T x_{n}\right),\left[d\left(x_{n-1}, T x_{n}\right)+d\left(x_{n}, T x_{n-1}\right)\right] / q\right\} \\
= & h\left(\tau_{n-1}\right) \cdot \tau_{n-1} .
\end{aligned}
$$

Case II. $\quad x_{n} \in P, x_{n+1} \in Q$.

$$
\begin{aligned}
d\left(x_{n}, x_{n+1}\right) & \leq d\left(x_{n}, x_{n+1}^{\prime}\right)=d\left(T x_{n-1}, T x_{n}\right) \\
& \leq h\left(\tau_{n-1}\right) \cdot \tau_{n-1} .
\end{aligned}
$$

Case III. $x_{n} \in Q, x_{n+1} \in P$. Since $x_{n} \in Q$ and is a convex combination of $x_{n-1}$ and $x_{n}^{\prime}$, it follows that $d\left(x_{n}, x_{n+1}\right) \leq \max .\left\{d\left(x_{n-1}, x_{n+1}\right), d\left(x_{n}^{\prime}, x_{n+1}\right)\right\}$. If $d\left(x_{n-1}, x_{n+1}\right) \leq$ 
$d\left(x_{n}^{\prime}, x_{n+1}\right)$, then $d\left(x_{n}, x_{n+1}\right) \leq d\left(x_{n}^{\prime}, x_{n+1}\right)=d\left(T x_{n-1}, T x_{n}\right) \leq h\left(\tau_{n-1}\right) \cdot \max \cdot\left\{\tau_{n-1} / 2\right.$, $\left.d\left(x_{n-1}, T x_{n-1}\right), d\left(x_{n}, T x_{n}\right),\left[d\left(x_{n-1}, T x_{n}\right)+d\left(x_{n}, T x_{n-1}\right)\right] / q\right\}=h\left(\tau_{n-1}\right) \cdot \max \cdot\left\{d\left(x_{n-1}\right.\right.$, $\left.\left.x_{n}^{\prime}\right),\left[d\left(x_{n-1}, x_{n+1}\right)+d\left(x_{n}, x_{n}^{\prime}\right)\right] / q\right\}$.

So, in the case where $d\left(x_{n-1}, x_{n}^{\prime}\right)$ is the maximum, we get:

$$
\begin{aligned}
d\left(x_{n}, x_{n+1}\right) & \leq h\left(\tau_{n-1}\right) \cdot d\left(x_{n-1}, x_{n}^{\prime}\right) \\
& \leq h\left(\tau_{n-1} \cdot h\left(\tau_{n-2}\right) \cdot \tau_{n-2} .\right.
\end{aligned}
$$

(by Case II, since $x_{n} \in Q$ implies that $x_{n-1} \in P$ ). On the other hand, if $\left[d\left(x_{n-1}, x_{n+1}\right)+\right.$ $\left.d\left(x_{n}, x_{n}^{\prime}\right)\right] / q$ is the maximum, we get:

$$
\begin{aligned}
d\left(x_{n}, x_{n+1}\right) & \leq h\left(\tau_{n-1}\right) \cdot\left[d\left(x_{n-1}, x_{n+1}\right)+d\left(x_{n}, x_{n}^{\prime}\right)\right] / q \\
& \leq h\left(\tau_{n-1}\right) \cdot\left[\tau_{n-1}+\tau_{n}+d\left(x_{n}, x_{n}^{\prime}\right)\right] / q \\
& =h\left(\tau_{n-1}\right) \cdot\left[d\left(x_{n-1}, x_{n}^{\prime}\right)+\tau_{n}\right] / q
\end{aligned}
$$

Therefore, $\left[1-h\left(\tau_{n-1}\right) / q\right] \cdot \tau_{n} \leq\left[h\left(\tau_{n-1}\right) / q\right] \cdot d\left(x_{n-1}, x_{n}^{\prime}\right)$

and thus

$$
\begin{aligned}
\tau_{n} \leq \frac{h\left(\tau_{n-1}\right)}{q-h\left(\tau_{n-1}\right)} \cdot d\left(x_{n-1}, x_{n}^{\prime}\right) & \\
& \leq h\left(\tau_{n-1}\right) \cdot d\left(x_{n-1}, x_{n}^{\prime}\right)
\end{aligned}
$$

Again, we conclude that:

$$
\tau_{n} \leq h\left(\tau_{n-1}\right) \cdot h\left(\tau_{n-2}\right) \cdot \tau_{n-2}
$$

Finally, we considew the possibility that $d\left(x_{n}^{\prime}, x_{n+1}\right)<d\left(x_{n-1}, x_{n+1}\right)$, here we have,

$$
\begin{aligned}
\tau_{n} \leq & d\left(x_{n-1}, x_{n+1}\right)=d\left(T x_{n-2}, T x_{n}\right) \\
\leq & h\left(s_{n-2}\right) \cdot \max \cdot\left\{s_{n-2} / 2, d\left(x_{n-2}, T x_{n-2}\right),\right. \\
& \left.d\left(x_{n}, T x_{n}\right),\left[d\left(x_{n-2}, T x_{n}\right)+d\left(x_{n}, T x_{n-2}\right)\right] / q\right\} .
\end{aligned}
$$

Note that

$$
\begin{aligned}
s_{n-2} / 2 & \leq\left[d\left(x_{n-2}, x_{n-1}\right)+d\left(x_{n-1}, x_{n}\right)\right] / 2 \\
& \leq \max \cdot\left\{\tau_{n-2}, \tau_{n-1}\right\} \\
& =\tau_{n-2} .
\end{aligned}
$$

So, we may conclude either,

$$
\tau_{n} \leq h\left(s_{n-2}\right) \cdot \tau_{n-2}
$$

or, in case the maximum of the right hand side of $\left(^{*}\right)$ is $\left[d\left(x_{n-2}, x_{n+1}\right)+d\left(x_{n-1}, x_{n}\right)\right] / q$, it follows that $d\left(x_{n-1}, x_{n+1}\right) \leq h\left(s_{n-2}\right) \cdot\left[d\left(x_{n-2}, x_{n-1}\right)+d\left(x_{n-1}, x_{n+1}\right)+d\left(x_{n}, x_{n+1}\right)\right] / q$, 
i.e., $\left[q-h\left(s_{n-2}\right)\right] \cdot d\left(x_{n-1}, x_{n+1}\right) \leq h\left(s_{n-2}\right) \cdot\left[1+h\left(\tau_{n-2}\right)\right] \cdot d\left(x_{n-2}, x_{n-1}\right)\left(\right.$ since $d\left(x_{n}, x_{n-1}\right)$ $\left.\leq d\left(x_{n-1}, x_{n}^{\prime}\right) \leq h\left(\tau_{n-2}\right) \cdot \tau_{n-2}\right)$.

Therefore, $d\left(x_{n-1}, x_{n+1}\right) \leq \frac{h\left(s_{n-2}\right)}{\left[1+h\left(s_{n-2}\right)\right]} \cdot\left[1+h\left(\tau_{n-2}\right)\right] \cdot \tau_{n-2}$ and thus, $\tau_{n} \leq \frac{h\left(s_{n-2}\right)}{\left[1+h\left(s_{n-2}\right)\right]}$. $\left[1+h\left(\tau_{n-2}\right)\right] \cdot \tau_{n-2}$. Now, if $s_{n-2} \geq \tau_{n-2}$, then $h\left(s_{n-2}\right) \leq h\left(\tau_{n-2}\right)$ and consequently, $\frac{h\left(s_{n-2}\right)}{1+h\left(s_{n-2}\right)} \leq \frac{h\left(\tau_{n-2}\right)}{1+h\left(\tau_{n-2}\right)}$. It follows:

$$
\tau_{n} \leq h\left(\tau_{n-2}\right) \cdot \tau_{n-2}
$$

On the other hand, if $s_{n-2}<\tau_{n-2}$, then $h\left(s_{n-2}\right) \geq h\left(\tau_{n-2}\right)$ and thus $1+h\left(s_{n-2}\right) \geq$ $1+h\left(\tau_{n-2}\right)$, or $\left(\left[1+h\left(\tau_{n-2}\right)\right] /\left[1+h\left(s_{n-2}\right)\right]\right) \leq 1$ and thus we get,

$$
\tau_{n} \leq h\left(s_{n-2}\right) \cdot \tau_{n-2}
$$

Finally, using the seven conclusions (A1)-(A7), we may conclude that for $n=2,3,4, \ldots$, we have

$$
\tau_{n}<\tau_{n-1}
$$

or

$$
\tau_{n}<\tau_{n-2}
$$

Step II. We will prove that the sequence $\left\{\tau_{n}\right\}_{n=0}^{\infty}$ converges to 0 . To do that, we consider two cases. In the first one we assume that $\left\{x_{n}\right\}$ has a subsequence $\left\{x_{n(k)}\right\}$ with the property that $x_{n(k)+1}$ and $x_{n(k)+2} \in P$. Here we consider the sequence $\tau_{n(k)}$. By (A) we observe that $\tau_{n(k)} \leq \tau_{n(k-1)+1}$ or $\tau_{n(k)} \leq \tau_{n(k-1)+2}$. Noting that $x_{n(k-1)+1}$ and $x_{n(k-1)+2} \in P$, it follows that $\tau_{n(k-1)+2}<\tau_{n(k-1)+1}<\tau_{n(k-1)}$. So, we may conclude that for $k \geq 2, \tau_{n(k)}<\tau_{n(k-1)}$ and thus $\tau_{n(k)} \rightarrow \tau$. We show that $\tau=0$. Observe that for $k=1,2,3, \ldots$, we have,

$$
\tau_{n(k+1)} \leq d\left(x_{n(k)+1}^{\prime}, x_{n(k)+2}^{\prime}\right)<\tau_{n(k)}
$$

and thus $\lim _{k \rightarrow \infty} d\left(x_{n(k)+1}^{\prime}, x_{n(k)+2}^{\prime}\right)=\tau$. If $\tau>0$, then $d\left(x_{n(k)+1}^{\prime}, x_{n(k)+2}^{\prime}\right) \leq h\left(\tau_{n(k)}\right)$. $\tau_{n(k)}$ and as $k \rightarrow \infty$ we obtain $\tau \leq h(\tau) \cdot \tau<\tau$. Contradiction. Moreover, for $j$ sufficiently large, $\exists k=k(j)$ such that $n(k) \leq j \leq n(k+1)$ and thus $0<\tau_{j} \leq \tau_{n(k)}$. Since $\tau_{n(k)} \rightarrow 0$, we conclude that $\lim _{j \rightarrow \infty} \tau_{j}=0$. In the second case, we assume that eventually the sequence $\left\{x_{n}\right\}$ cannot have two consecutive points that are in $P$, ie., $\exists$ a positive integer $N$ such that for every $n \geq N$, if $x_{n} \in P$ then $x_{n+1} \in Q$, Assume that $x_{n}(i) \in Q$ for $i=1,2,3, \ldots$, where $n(i)+2=n(i+1)$ and $n(i)-2=n(i-1)$, and consider the sequence $\left\{\tau_{n(i)}\right\}$. Note that $\tau_{n(i)}$ is convergent, and suppose that $\tau_{n(i)} \rightarrow \tau$. By (Observation 2.1, [2]), we may assume that $\exists$ a subsequence of $\left\{x_{n(i)}\right\}$ denoted by $x_{n(t)}$ such that either,

(B) for $t=1,2,3, \ldots, \tau_{n(t)} \leq d\left(x_{n(t)+1}, x_{n(t)}^{\prime}\right)$, or

(C) for $t=1,2,3, \ldots, \tau_{n(t)} \leq d\left(x_{n(t)+1}, x_{n(t)-1}\right)$.

If Case (B) occurs, then by (A3) and (A4) we have: 
(D) $\tau_{n(t)}<d\left(x_{n(t)-1}, x_{n(t)}^{\prime}\right)<\tau_{n(t)-2}$, it follows that $\lim \tau_{n(t)}=\lim d\left(x_{n(t)-1}^{\prime}, x_{n(t)}^{\prime}\right)=$ $\tau=0$, and $\lim \tau_{n}=0$.

Finally, we consider the possibility that (C) occurs. Then by (A5), (A6) and (A7), we may assume that for $t=1,2,3, \ldots$, we have,

$$
\tau_{n(t)} \leq h\left(\tau_{n(t)-2}\right) \cdot \tau_{n(t)-2}
$$

or

$$
\tau_{n(t)} \leq h\left(s_{n(t)-2}\right) \cdot \tau_{n(t)-2} .
$$

In the case (E1) occurs, as $t \rightarrow \infty$, we get $\tau \leq h(\tau) \cdot \tau<\tau$, which is absurd, and thus we conclude that $\tau=0$. On the other hand if (E2) occurs, without loss of generality, and since $\left\{s_{n(t)-2}\right\}$ is bounded, we may assume that $s_{n(t)-2} \rightarrow \rho$. If $\rho>0$, then as $t \rightarrow \infty$, we get $\tau \leq h(\rho / 2) \cdot \tau<\tau$. Contradiction. To show that $\tau=0$, we note that:

$$
\tau_{n(t)-2}-d\left(x_{n(t)-2}, x_{n(t)}\right) \leq d\left(x_{n(t)-1}, x_{n(t)}\right)<d\left(x_{n(t)-1}, x_{n(t)}^{\prime}\right)<\tau_{n(t)-2} .
$$

Hence $\lim d\left(x_{n(t)-1}^{\prime}, x_{n(t)}^{\prime}\right)=\tau$ and we may conclude as we did in the previous two cases that $\lim _{n \rightarrow \infty} \tau_{n}=0$. So we have:

$$
\lim _{n \rightarrow \infty} \tau_{n}=0 .
$$

Step III. We prove that $\left\{x_{n}\right\}$ is a Cauchy sequence. For if it is not Cauchy, then by well-ordering principle there exists $\epsilon>0$ and two subsequences $\{p(n)\},\{l(n)\}$ such that for every $n=0,1,2,3, \ldots$, we find that $p(n)>l(n)>n, d\left(x_{p(n)}, x_{l(n)}\right) \geq \epsilon$ and $d\left(x_{p(n)-1}, x_{l(n)}\right)<\epsilon$. Put $g_{n}=d\left(x_{p(n)}, x_{l(n)}\right)$. For each $n \geq 0$, we have:

$$
\begin{aligned}
\varepsilon \leq g_{n} & \leq d\left(x_{p(n)-1}, x_{p(n)}\right)+d\left(x_{p(n)-1}, x_{\ell(n)}\right) \\
& <\tau_{p(n)-1}+\varepsilon .
\end{aligned}
$$

Since $\tau_{n} \rightarrow 0$, it follows that $g_{n} \rightarrow \varepsilon$. It has been shown in details in Assad [1] that (F) allows us to conclude that:

$$
\begin{aligned}
& \lim d\left(x_{p(n)+1}, x_{\ell(n)-1}\right)=\lim d\left(x_{p(n)-1}, x_{\ell(n)+1}\right) \\
= & \lim d\left(x_{p(n)+1}, x_{\ell(n)+1}\right)=\lim d\left(x_{p(n)+1}, x_{\ell(n)}\right) \\
= & \lim d\left(x_{p(n)}, x_{\ell(n)-1}\right)=\lim d\left(x_{p(n)-1}, x_{\ell(n)-1}\right) \\
= & \lim d\left(x_{p(n)}, x_{\ell(n)+1}\right)=\lim d\left(x_{p(n)-1}, x_{\ell(n)}\right)=\varepsilon .
\end{aligned}
$$

Next, we consider the following four possibilites :

$$
x_{p(n)+1}, \in P \text { and } x_{\ell(n)+1} \in P \text {, then }
$$




$$
\begin{aligned}
d\left(x_{p(n)+1}, x_{\ell(n)+1}\right)= & d\left(T x_{p(n)}, T x_{\ell(n)}\right) \\
\leq & h\left(g_{n}\right) \cdot \max \cdot\left\{g_{n} / 2, \tau_{p(n)}, \tau_{\ell(n)},\right. \\
& {\left.\left[d\left(x_{p(n)}, T x_{\ell(n)}\right)+d\left(x_{\ell(n)}, T x_{p(n)}\right)\right] / q\right\} . }
\end{aligned}
$$

$$
x_{p(n)+1} \in P \text { and } x_{\ell(n)+1} \in Q \text {, then } x_{\ell(n)} \in P \text { and }
$$

$$
\begin{aligned}
d\left(x_{p(n)+1}, x_{\ell(n)}\right)= & d\left(T x_{p(n)}, T x_{\ell(n)-1}\right) \\
\leq & h\left(d\left(x_{p(n)}, x_{\ell(n)-1}\right)\right) \cdot \max \cdot\left\{d\left(x_{p(n)}, x_{\ell(n)-1}\right) / 2, \tau_{p(n)}, \tau_{\ell(n)-1},\right. \\
& {\left.\left[g_{n}+d\left(x_{\ell(n)-1}, x_{p(n)+1}\right)\right] / 1+2 h\left(d\left(x_{p(n)}, x_{\ell(n)-1}\right)\right)\right\} . }
\end{aligned}
$$

$$
x_{p(n)+1} \in Q \text { and } x_{\ell(n)+1} \in P \text {, then } x_{p(n)} \in P \text { and }
$$

$$
\begin{aligned}
d\left(x_{p(n)}, x_{\ell(n)+1}\right)= & d\left(T x_{p(n)-1}, T x_{\ell(n)}\right) \\
\leq & h\left(d\left(x_{p(n)-1}, x_{\ell(n)}\right)\right) \cdot \max \cdot\left\{d\left(x_{p(n)-1}, x_{\ell(n)}\right) / 2, \tau_{p(n)-1}, \tau_{\ell(n)},\right. \\
& {\left.\left[g_{n}+d\left(x_{p(n)-1}, x_{\ell(n)+1}\right)\right] / 1+2 h\left(d\left(x_{p(n)-1}, x_{\ell(n)}\right)\right)\right\} . }
\end{aligned}
$$

(G4)

$$
x_{p(n)+1} \in Q \text { and } x_{\ell(n)+1} \in Q \text {, then } x_{p(n)} \text { and } x_{\ell(n)} \in P \text { and, }
$$

$$
\begin{aligned}
d\left(x_{p(n)}, x_{\ell(n)}\right)= & d\left(T x_{p(n)-1}, T x_{\ell(n)-1}\right) \\
\leq & h\left(d\left(x_{p(n)-1}, x_{\ell(n)-1}\right)\right) \cdot \max \cdot\left\{d\left(x_{p(n)-1}, x_{\ell(n)-1}\right) / 2, \tau_{p(n)-1}, \tau_{\ell(n)-1},\right. \\
& {\left.\left[d\left(x_{p(n)-1}, x_{\ell(n)}\right)+d\left(x_{\ell(n)-1}, x_{p(n)}\right)\right] / 1+2 h\left(d\left(x_{p(n)-1}, x_{\ell(n)-1}\right)\right)\right\} . }
\end{aligned}
$$

Each of these four Cases: (G1), (G2), (G3) and (G4) leads to the conclusion that $\varepsilon \leq \frac{2 h(\varepsilon / 2)}{1+2 h(\varepsilon / 2)} \cdot \varepsilon<\varepsilon$ as $n \rightarrow \infty$, which is absurd. Therefore, we conclude that the sequence $\left\{x_{n}\right\}$ is a Cauchy sequence, and by completeness of $X$, we conclude that the sequence converges to a point in $K$. Let $\lim _{n \rightarrow \infty} x_{n}=z$.

Finally, we will show that $z$ is the unique fixed point of $T$. Choose a subsequence $\left\{x_{b(n)}\right\}_{n=0}^{\infty}$ of $\left\{x_{n}\right\}_{n=0}^{\infty}$ such that $x_{b(n)+1} \in P$ for all $n=0,1,2, \cdots$ Observe that $\lim _{n \rightarrow \infty} x_{b(n)+1}=\lim _{n \rightarrow \infty} x_{n}=z$ and by continuity of $T$ we also have $\lim _{n \rightarrow \infty} x_{b(n)+1}=$ $\lim _{n \rightarrow \infty} T x_{b(n)}=T z$. Therefore we obtain that $z=T z$. If $T$ has two distinct fixed points $x, y \in K$, then

$$
\begin{aligned}
d(x, y)= & d(T x, T y) \\
\leq & h(d(x, y)) \cdot \max \cdot\{d(x, y) / 2, d(x, T x), d(y, T y), \\
& {[d(x, T y)+d(y, T x)] / 1+2 h(d(x, y))\}, }
\end{aligned}
$$

and thus $d(x, y) \leq \frac{2 h(d(x, y))}{1+2 h(d(x, y))} \cdot d(x, y)<d(x, y)$, a contradiction. Thus the proof is completed.

The following result follows immediately from the Theorem.

Corollary. Let $X$ be a Banach space, $K$ a nonempty closed subset of $X, T: K \rightarrow X$ a continuous mapping satisfying the condition on $K$,

(H) for all $x \neq y, x, y \in K$ :

$$
d(T x, T y) \leq h(d(x, y)) \cdot \max \cdot\{d(x, T x), d(y, T y)\} .
$$

If $T$ has property (a), then $T$ has a unique fixed point. 


\section{References}

[1] N.A. Assad," On some monself nonlinear contractions," Math. Japonica 33, No.1 (1988), 17-26.

[2] N.A. Assad, "On some nonself mappings in Banach spaces," Math. Japonica 33, No.4 (1988),
501-515.

[3] N.A. Assad, "A fixed point theorem in Banach space," to appear.

[4] L.E. Blumenthal, Theory and applications of distance geometry, Oxford, 1953.

[5] B.E. Rhoades, "A fixed point theorem for some nonself mappings," Math. Japonica 23, No.4 (1978),
$457-459$.

[6] M.S. Khan, M. Swaleh, and S. Sessa, "Fixed point theorems by altering distances between the points," Bull. Austral. Math. Sco., 30 (1984), 1-9.

Department of Mathematics, Birzeit University, Birzeit, P.O. BOX 14, West Bank, Via Israel. 